\title{
Colonic Ulceration Caused by Administration of Loxoprofen Sodium
}

\author{
Hiroshi NaKase****, Toshinao ITANI**, Jun Mimura*, Toshihiko KaWASAKI*, Hideshi Komori*, \\ Kimio HaShimoto** and Tsutomu CHIBA***
}

\begin{abstract}
A 54-year-old female with chronic headache was admitted to our hospital because of hematochezia. She had routinely taken loxoprofen sodium because of severe headache. Emergent colonoscopic examination revealed ulceration of the cecum. After administration of loxoprofen sodium was discontinued and administration of sulfasalazine was initiated, her intestinal bleeding subsided. Two months after discontinuation of loxoprofen sodium, the colonoscopic examination revealed scar formation at the site of cecal ulceration. In this case, it was conceivable that the administration of loxoprofen sodium might have induced colonic ulceration.
\end{abstract}

(Internal Medicine 38: 249-251, 1999)

Key words: non-steroidal anti-inflammatory drugs (NSAIDs), sulfasalazin, colonoscopy

\section{Introduction}

Non-steroidal anti-inflammatory drugs (NSAIDs) are one of the classes of drugs which have been widely utilized to date. However, reports on the side effects of NSAIDs have increased with their widespread use. In particular, gastroduodenal mucosal damage is well known as a complication of treatment using NSAIDs. For example, massive hemorrhage from gastric and duodenal ulcers or perforation during NSAID treatment have been reported (1-4). Recent studies have shown that long-term administration of NSAIDs results in inflammation of the small intestine and colon $(5,6)$. The pathogenesis of the inflammation is not completely understood but it is thought to involve several interacting factors.

Loxoprofen sodium is one of the NSAIDs, which has been widely used in Japan. In this report, we describe a case of colonic ulceration induced by administration of loxoprofen sodium.

For editorial comment, see also p 219.

\section{Case Report}

The patient, a 54-year-old female had, a history of chronic headache. She had taken loxoprofen sodium once or twice per day for a period of one year because of severe headache. On
March 18, 1995, she suddenly noticed hematochezia but it was not accompanied by abdominal pain. Because the hematochezia continued, she came to the emergency room of Nishi-Kobe Medical Center. Physical examination revealed no abnormalities. Laboratory test revealed the following: hemoglobin and hematocrit, $9.8 \mathrm{~g} / \mathrm{dl}$ and $31.2 \%$, respectively; white blood cell count, $3,000 / \mu \mathrm{l}$; platelet count, $1.56 \times 10^{5} / \mu 1$; total protein 6.0 $\mathrm{g} / \mathrm{dl}$; serum asparate transaminase, $16 \mathrm{IU} / \mathrm{l}$; serum alanine transaminase, $9 \mathrm{IU} / \mathrm{l}$; blood urea nitrogen (BUN), $7 \mathrm{mg} / \mathrm{dl}$; and creatinine, $0.8 \mathrm{mg} / \mathrm{dl}$. A lymphocyte stimulating test for loxoprofen sodium was negative. Viral and bacterial cultures of samples of her blood, urine, sputum and stool were negative. On the same day, endoscopic examination was performed. An upper endoscopic examination revealed no lesion which seemed to be bleeding. Colonoscopic examination revealed ulceration of the cecum (Fig. 1A). Small intestinal radiography showed no abnormalities. Based on these findings, we concluded that the hematochezia was due to colonic ulceration. Microscopic expamination of biopsy specimen revealed nonspecific inflammation (Fig. 2). Histological and bacteriological examinations excluded Crohn's disease, vascular lesions and infectious disease including tuberculosis. Administration of loxoprofen sodium was discontinued and administration of sulfasalazine was initiated. Following discontinuation of loxoprofen sodium treatment, her intestinal bleeding subsided. Two months after discontinuation of loxoprofem sodium, a colonoscopic examination was performed. Endoscopic findings revealed scar forma-

From *the Division of Gastroenterology, Nishi-Kobe, Medical Center, Kobe, **the Division of Pathology, Nishi-Kobe, Medical Center, Kobe and ***the Division of Gastroenterology, Department of Internal Medicine, Postgraduate School of Medicine, Kyoto University, Kyoto

Received for publication January 26, 1998; Accepted for publication November 16, 1998

Reprint requests should be addressed to Dr. Hiroshi Nakase, the Division of Gastroenterology, Department of Internal Medicine, Postgraduate School of Medicine, Kyoto University, 54 Syogoin Kawara-cho, Sakyo-ku, Kyoto 606-8397 

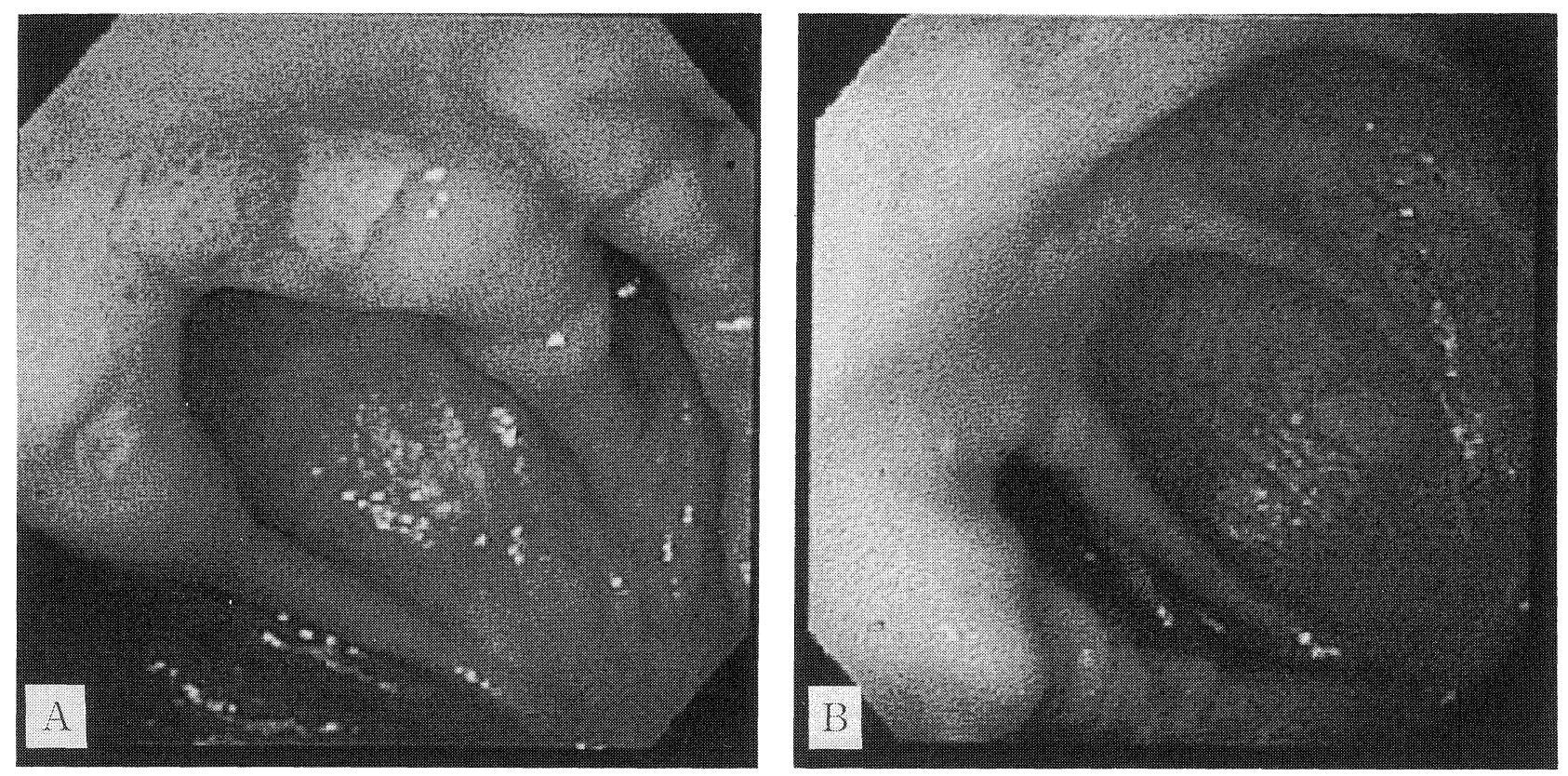

Figure 1. Endoscopic appearance of cecum. (A) Endoscopic examination carried out at admission demonstrating two ulcers with regenerated epitheliums. (B) Endoscopic examination demonstrating scar formation at the sites of the ulcers two months after discontinuation of loxoprofen sodium treatment.

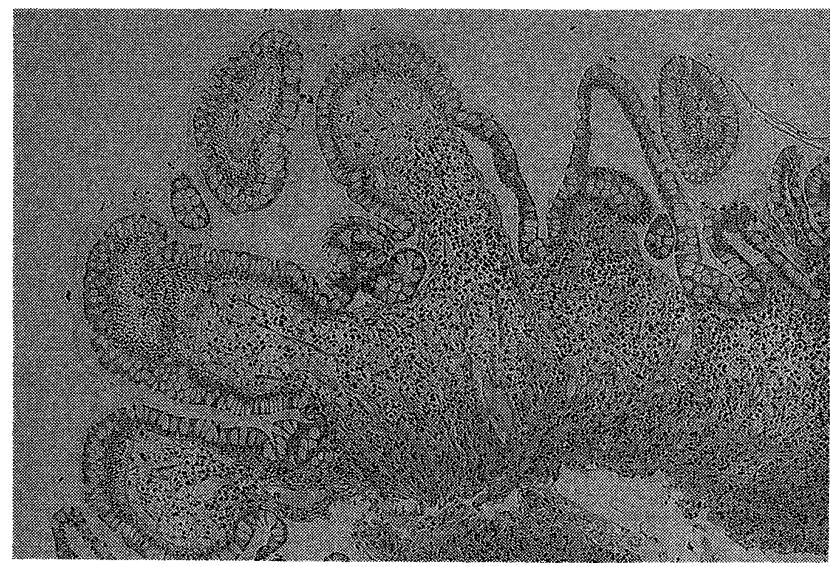

Figure 2. Histology of a biopsy specimen from the margin of cecal ulcer. The low power view demonstrates non-specific inflammation (HE stain, $\times \mathbf{4 0}$ ).

tion at the site of cecal ulceration (Fig. 1B). At present, this patient usually takes eperisone (Myonal) which is a muscle relaxant. She takes acetaminophen $(0.5 \mathrm{~g})$ and sulfasalazine $(500 \mathrm{mg})$ only when she complains of severe headache. Since she discontinued loxoprofen sodium treatment, she has not experienced hematochezia.

\section{Discussion}

Ulceration of the upper gastrointestinal tract as a result of
NSAID treatment is well known. Recently, the side effects of NSAIDs on the distal small bowel and colon are increasingly being reported (7-13). We investigated a case of hematochezia due to cecal ulceration. In general, with reference to the differential diagnosis of cecal ulceration, simple ulcer, Behçet's disease, colonic tuberculosis, and Crohn's disease, are well known. In this case of hematochezia, blood, histological and bacteriological examination ruled out infectious disease (tuberculosis, cytomegalovirus, herpes virus, etc.) and Crohn's disease. The fact that the patient did not present with recurrent oral ulcer or genital ulceration or uveitis ruled out Behçet's disease.

It is very difficult to distinguish cecal ulceration induced by NSAIDs from simple ulceration. However, in this case, discontinuation of loxoprofen sodium rapidly resulted in disapperance of the hematochezia. Cecal ulceration has not recurred after the patient discontinued taking loxoprofen sodium. Although the lymphocyte stimulating test for loxoprofen sodium was negative, the clinical course of the hematochezia strongly indicated that this ulceration was induced by NSAIDs.

The mechanism of NSAID-induced colonic ulceration may be related to the inhibition of intestinal prostaglandin synthesis. In the gut, prostaglandins have cytoprotective actions and various prostaglandins are synthesized by human colonic tissue. NSAIDs inhibit cyclo-oxygenase, thereby reducing prostaglandin production. Decreased prostaglandin synthesis may cause an increase in intestinal permeability, which would destroy local defense systems and allow entrance of bacteria into the mucosa, resulting in inflammatory infiltration. In addition, NSAIDs may divert arachidonic acid toward the lipoxygenase pathway with resultant production of leukotrienes and free radi- 
cals, thereby causing inflammation and tissue injury in the lower gut $(4,7)$.

Treatment of NSAID-induced colitis is reported to require only discontinuation of the offending agent. In this case, we treated our patient not only by rapidly discontinuing loxoprofen sodium administration but also by initiating sulfasalazine administration to accelerate the healing of colonic ulceration. Sulfasalazine inhibits the synthesis of lipoxygenase products and thromboxane B2 while enhancing the synthesis of prostaglandin E2 (14). Bjarnason et al reported that there was a reduction in intestinal inflammation of patients with rheumatoid arthritis by administration of sulfasalazine $(15,16)$. Therefore, in the case of NSAID-induced colitis, we must take into consideration that administration of sulfasalazine is one therapy.

Recently, Picot and colleagues reported the finding of two subtypes of cyclooxygenases (COX1 and COX2) (17). COX1 is constitutive and COX 2 is inducible. The former is found in platelets producing thromboxane 2 in the endothelium, producing prostacyclin in the stomach and producing prostaglandin E2, in the kidney. The latter is found in macrophages and in other cells that produce proteases, prostaglandins, and other inflammatory mediators. Therefore, if NSAIDs which inhibit COX 2 only are developed, NSAID-induced side effects on the gut may be decreased. With the expected increase in the use of NSAIDs in the future, reports of NSAID-induced gastroenteropathy and complications thereof may increase. As we found in the present case, total colonscopy was very useful for examination of a patient complaining of hematochezia. Thus, in a patient with such a complaint of hematochezia, who has been receiving long-term administration of NSAIDs, it is advisable to carry out a colonoscopic examination to examine for the presence of colonic ulcer or small intestinal ulcer.

\section{References}

1) Collier DS, Pain JA. Non-steroidal anti-inflammatory drugs and peptic ulcer perforation. Gut 26: 359-363, 1985.

2) Matthewson K, Pugh S, Northfield TC. Which peptic ulcer patients bleed? Gut 29: 70-74, 1988.

3) O'Brien JD, Burnham WR. Bleeding from peptic ulcers and use of nonsteroidal anti-inflammatory drugs in the Romford area. Br Med J (Clin Res Ed) 291: 1609-1610, 1985.

4) Hirschowitz BI. Nonsteroidal antiinflammatory drugs and the gastrointestinal tract. Gastroenterologist 2: 207-223, 1994.

5) Bjarnason I, Zanelli G, Smith T, et al. Nonsteroidal antiinflammatory druginduced intestinal inflammation in humans. Gastroenterology 93: 480489, 1987.

6) Cappell MS, Simon T. Colonic toxicity of administered medications and chemicals. Am J Gastroenterol 88: 1684-1699, 1993.

7) Gibson GR, Whitacre EB, Ricotti CA. Colitis induced by nonsteroidal anti-inflammatory drugs. Report of four cases and review of the literature. Arch Intern Med 152: 625-632, 1992.

8) Gizzi G, Villani V, Brandi G, Paganelli GM, Di Febo G, Biasco G. Anorectal lesions in patients taking suppositories containing non-steroidal antiinflammatory drugs (NSAIDs). Endoscopy 22:146-148, 1990.

9) Hakozaki Y, Shirahama T, Kotoh M, et al. A case of non-steroidal antiinflammatory drug-induced small intestinal ulcer and stricture. Gastroenterol Endosc 35: 1363, 1993 (Abstract).

10) Matsuhashi N, Yamada A, Hiraishi M, et al. Multiple stricture of the small intestine after long-term nonsteroidal anti-inflammatory drug therapy. Am J Gastroenterol 87: 1183-1186, 1992.

11) Whitcomb DC, Martin SP, Trellis DR, Evans BA, Becich MJ. 'Diaphragmlike' stricure and ulcer of the colon during diclofenac treatment. Arch Intern Med 152: 2341-2343, 1992.

12) Bjarnason I, Price AB, Zanelli G, et al. Clinicopathological features of nonsteroidal antiinflammatory drug-induced small intestinal strictures. Gastroenterology 94: 1070-1074, 1988.

13) Bjarnason I, Hayllar J, MacPherson AJ, Russell AS. Side effects of nonsteroidal anti-inflammatory drugs on the small and large intestine in humans. Gastroenterology 104: 1832-1847, 1993.

14) Hawkey CJ, Boughton-Smith NK, Whittle BJ. Modulation of human colonic arachidonic acid metabolism by sulfasalazine. Dig Dis Sci 30: 1161$1165,1985$.

15) Bjarnason I, Hopkinson N, Zanelli G, et al. Treatment of non-steroidal anti-inflammatory drug induced enteropathy. Gut 31: 777-780, 1990.

16) Bjarnason I, Hopkinson N, Zanelli G, et al. Treatment of NSAID induced small intestimal inflammation. Gut 29: A1434, 1988 (Abstract).

17) Picot $D$, Loll PJ, Garavito RM. The X-ray crystal structure of the membrane protein prostaglandin $\mathrm{H}_{2}$ synthase-1. Nature 367: 243-249, 1994. 